\title{
Evaluación de la mezcla de mercadeo digital en las plataformas de venta virtual de autoservicios en Colombia
}

\section{Evaluation of the Digital Marketing mix of Virtual Self- Service Sales Platforms in Colombia}

Paula Andrea Mejía-Henao'; Yeimy Viviana Marín-Chaves²

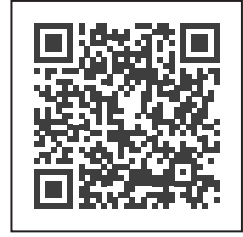

\section{Palabras claves:}

mercadeo digital, plataformas virtuales, calidad de sitios web.

Articulo de investigación:

Fecha de recepción:

05/05/2020

Fecha de aceptación: $30 / 07 / 2020$

Esta publicación se encuentra bajo licencia:

Creative Commons ReconocimientoNoComercialSinObraDerivada 4.0 Internacional

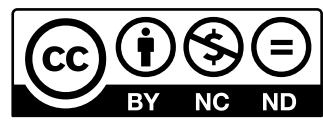

\section{Resumen}

Esta investigación aborda la evaluación y el análisis de los componentes de la mezcla de mercadeo digital (producto, precio, plaza, promoción y plataforma [5 P]) plasmada en las plataformas de venta virtual de autoservicios en Colombia. En Colombia es evidente la falta de dinamismo para aprovechar todas las bondades que el comercio electrónico y el mercadeo digital pueden ofrecer a las empresas, que pueden aportar a su desarrollo y crecimiento. Esta investigación pretende identificar, clasificar, evaluar y analizar la mezcla de mercadeo digital de las plataformas de venta virtual de los autoservicios existentes en Colombia. La metodología de investigación desarrollada es de enfoque cuantitativo, el alcance es descriptivo, el diseño es de carácter no experimental transversal, la técnica utilizada es la de análisis de contenido y el instrumento para la recolección de información es el cuestionario. Esta investigación encontró que la gran mayoría de las plataformas de venta virtual utilizan variadas

1 Administradora de empresas y magíster en Mercadeo. Coordinación de autoevaluación, programa académico de Administración de Empresas, Facultad de Ciencias Económicas y Administrativas, Universidad de San Buenaventura, sede Bogotá, Colombia. pamejia444@hotmail.com, ORCID: https://orcid.org/0000-0003-4525-1893

2 Administradora de empresas y magíster en Sistemas Integrados de Gestión. Dirección del programa académico de Administración de Empresas, Facultad de Ciencias Económicas y Administrativas, Universidad de San Buenaventura, sede Bogotá, Colombia. marinvivic@hotmail.com, ORCID: https://orcid.org/0000-0002-1142-3370 


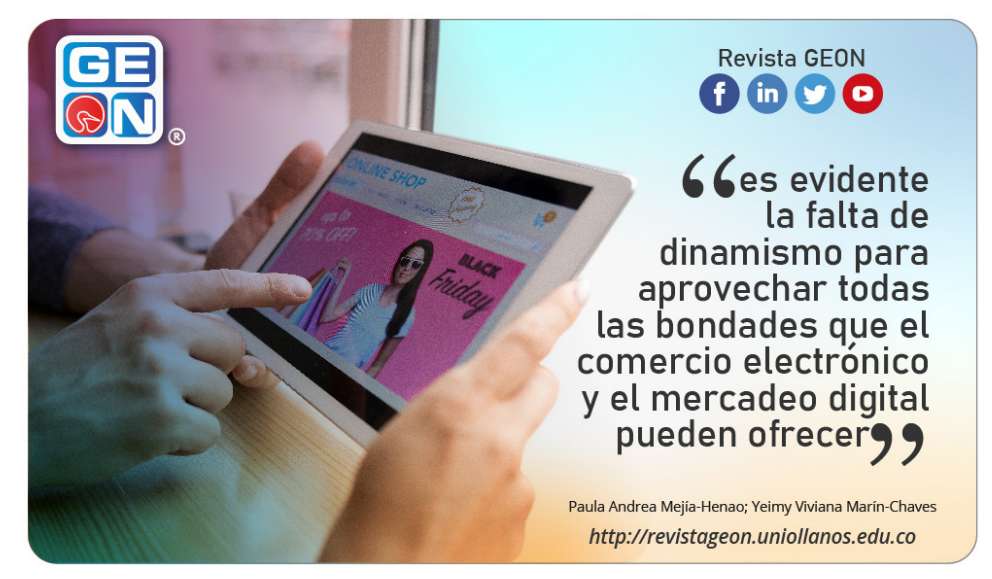

herramientas del mercadeo digital que crean una experiencia única y agradable para el cliente, en consonancia con lo expresado por la teoría de esta rama de la disciplina, en cuanto a su estructuración (5 P) y los modelos para la evaluación de sitios web. La gran mayoría de las plataformas de venta virtual de autoservicios en Colombia analizadas presentan una estructuración muy pertinente en cuanto a la mezcla de marketing digital y dan cuenta de la solución a las problemáticas existentes hace algunos años, lo que hace que esta herramienta se constituya en un impulso comercial para estas empresas.

Palabras clave: mercadeo digital, plataformas virtuales, calidad de sitios web.

\section{Abstract}

This research addresses the evaluation and analysis of the components of the digital marketing mix (product, price, place, promotion, and platform [5P]) embodied in the virtual self-service sales platforms in Colombia. In Colombia it is evident the lack of dynamism to take advantage of all the

Cómo citar este artículo / Toreference this article:

Mejia Henao, P. A., \& Marín Chaves, Y. V. (2020). Evaluación de la mezcla de mercadeo digital en las plataformas de venta virtual de autoservicios en Colombia. Revista GEON (Gestión, Organizaciones Y Negocios), 7(2). https://doi. org/10.22579/23463910.212 benefits that e-commerce and digital marketing can offer to the companies, which can contribute to their development and growth. This research intends to identify, classify, evaluate, and analyze the digital marketing mix of the virtual sales platforms of the self-services existing in Colombia. The research methodology developed is of a quantita- 
Cómo citar este artículo / Toreference this article:

Mejia Henao, P. A., \& Marín Chaves, Y. V. (2020). Evaluación de la mezcla de mercadeo digital en las plataformas de venta virtual de autoservicios en Colombia. Revista GEON (Gestión, Organizaciones Y Negocios), 7(2). https://doi. org/10.22579/23463910.212 tive approach, the scope is descriptive, the design is of a non-experimental transversal nature, the technique used is that of content analysis and the instrument for the collection of information is the questionnaire. This research found that the vast majority of virtual sales platforms use different digital marketing tools that create a unique and enjoyable experience for the customer, in line with the theory of this branch of the discipline, in terms of its structure (5 P) and models for evaluating websites. The vast majority of self-service virtual sales platforms in Colombia analyzed present a very pertinent structure in terms of the digital marketing mix and give an account of the solution to the problems that existed some years ago, making this tool a commercial boost for these companies.

Keywords: Digital marketing, virtual platforms, website quality.

Códigos JEL: M Administración de Empresas y Economía de la Empresa, Marketing, Contabilidad, Economía del personal, M3 Marketing y Publicidad, M31 Marketing.

\section{Introducción}

Esta investigación presenta información relacionada con la evaluación de la estructuración de la mezcla de mercadeo digital, conformada por producto, precio, plaza, promoción y plataforma (5 P), disponible en los sitios virtuales de cuatro establecimientos de autoservicios ubicados en territorio colombiano. Sus objetivos son identificar, clasificar, evaluary analizar la estructuración de esta.

En Colombia, las transacciones del comercio electrónico han venido promo- viendo la comercialización y la venta de productos/servicios a nivel nacional e internacional, en donde el mercadeo digital cobra gran importancia y es aliado estratégico para su impulso, pues se constituye en un sistema alternativo importante (Observatorio eCommerce Colombia, 2019), que representó el 8,5\% del PIB nacional para el año 2018 (La República, 2019).

El Departamento Nacional de Planeación ha identificado los principales problemas en el comercio electrónico que se relacionan con el mercadeo digital, a saber: a) la falta de utilización 
de estrategias de mercadeo digital; b) la desconfianza en relación con la seguridad y la privacidad en internet; y c) la baja oferta de contenido web (Consejo Nacional de Política Económica y Social, 2009).

La investigación está estructurada de la siguiente manera: a) establecimiento del marco teórico del mercadeo digital y su conformación, y modelos de evaluación de sitios web; b) descripción de la metodología utilizada para su desarrollo; c) resultados en relación con la mezcla de mercadeo digital encontrada en las plataformas de venta virtual; y d) conclusiones más relevantes.

\section{Contexto teórico}

Kannan y Li (2016) definen el mercadeo digital como "un proceso adaptable y habilitado por la tecnología mediante el cual las empresas colaboran con los clientes y los asociados para crear, comunicar, entregar y mantener [...] valor [...]" (pp. 4-5). El Instituto de Mercadeo Digital (DMI), citado por Bizhanova, Mamyrbekov, Umarov, Orazymbetova y Khairullaeva (2019), lo percibe como un medio para el establecimiento de las relaciones duraderas con el cliente y Selman (2017), citado por Acosta y Martínez (2018), la concibe como estrategia que garantiza la visita del cliente al sitio web.

Borden (1964) y McCarthy (1964), citados por Constantinides (2002), propusieron la mezcla de mercadeo tradicional compuesta por las 4 P (Azadi \& Rahimzadeh). Autores como Constantinides (2002), Zwass (1996), citado por Allen y Fjermestad (2002), Kannan y Li (2016) y Chaffey y Ellis-Chadwick (2014) definen un marco para la estructuración del mercadeo digital compuesta por: a) producto (tangible o intangible, personalizado, relacionado con la marca); b) precio (costo de fácil acceso y transacción, modelo para fijación); c) plaza (sitio web, punto de venta y de interacción, canales de distribución); y d) promoción (contenido para informar bondades del producto, persuadir al cliente y posicionar la marca). Chaffey (2000), citado por Constantinides (2002), toma el enfoque tradicional de la mezcla de mercadeo como punto de partida de la comercialización virtual e incluye una quinta $P$, con la que se relaciona el cliente en su interacción durante el proceso de compra, representada por la plataforma (lugar en la virtualidad donde ocurre la experiencia del cliente).

La Asociación Española de Comercio Electrónico y Marketing Relacional (AECEM) indica los principales elementos que deben estar incorporados en los sitios web; estos son: catálogo de productos/servicios, carrito de compras, dispositivos de promoción, motor de búsqueda, medios de pago, precios, mecanismos de logística y distribución, información organizacional y área del usuario (AECEM, 2009).

Existen variados modelos de evaluación de sitios web que consideran diferentes enfoques para su análisis. Estos están relacionados con: a) los elementos claves representado por los "factores de éxito en los sitios web" 
de Park (2007); b) los factores clave de calidad en sitios web eficientes representados por el "índice cuantitativo de calidad Web (ICCW)"de González (2013), el "WebQual 4.0" de Barnes (2002), el "índice de evaluación web (IEW)" de Miranda (2005) y el "método cuantitativo para la evaluación de calidad de sitio web (WebQEM)" de Olsina (2002); c) los niveles de calidad en el servicio al cliente en la web representados por el "WEBQUAL" de Liacono (2014) y el "E-SERVQUAL" de Zeithaml (2002); y d) la ingeniería web representada por el "modelo de calidad para sitios web" de Polillo (2011), la norma de calidad ISO/IEC 25010 (Portal ISO 25000, 2016) y la "Norma de calidad ISO/IEC 9126" (Universidad Nacional Abierta y a Distancia [UNAD], 2016). Estos modelos revisan principalmente variables como: usabilidad, accesibilidad, navegabilidad, funcionalidad, fiabilidad, confiabilidad, interactividad, intuitividad, velocidad, seguridad y privacidad, arquitectura, eficiencia de desempeño, calidad SEO del dominio, popularidad web, coherencia en diseño, estética, atractivo visual y emocional, calidad del contenido, comprensión, multicanalidad, contacto, comunidad, servicio al cliente, satisfacción, personalización, cumplimiento y capacidad de respuesta.

\section{Materiales y métodos}

La presente investigación posee las siguientes características (Hernández, 2014): a) enfoque de corte cuantitativo, debido a que los datos recolectados están basados en mediciones numéricas, y b) alcance de corte descriptivo sustentado en el interés por identificar y describir los diferentes elementos y características relacionadas con la mezcla de mercadeo digital en las plataformas de venta virtual de autoservicios.

El diseño de investigación es de corte no experimental transversal sustentado en que las diferentes variables (independientes) que son observadas en las plataformas de venta online son analizadas en un único momento, sin ser manipuladas de ninguna manera. La técnica de investigación utilizada para analizar las plataformas de venta virtual es la de análisis de contenido, la cual hace parte del campo de la observación.

El análisis de contenido (Andréu, 2018) es una metodología utilizada para la interpretación de textos en las diferentes maneras en que estos se puedan encontrar (escritos, pinturas, filmaciones, entre otras) y en las distintas formas en que estos se puedan tomar (informes, entrevistas, discursos, videos, sitios web, entre otras).

El instrumento estandarizado de medición, en este caso, el cuestionario, será construido a partir de las teorías relacionadas con la mezcla de mercadeo digital y con los modelos de evaluación de plataformas de venta virtual.

La población de estudio está conformada por los establecimientos que cumplan con las siguientes características: a) autoservicios (el cliente escoge los productos y paga al finalizar la compra); b) almacenes de cadena con presencia física nacional; c) venta al por menor; d) variedad de bienes 
de consumo (alimentos y uso doméstico); e) medianas superficies; y f) plataforma online propia. La totalidad de los autoservicios que cuentan con las características anteriormente descritas, a noviembre de 2017, son³: Fontana.com, Kolmena.com, Mandala. com y Dominico.com.

\section{Resultados}

Las categorías, subcategorías y variables que se estructuraron y analizaron en la mezcla de mercadeo digital presente en las plataformas de venta virtual son:

a. Producto: ordenación, descripción concreta y completa, visualización, desarrollo de marca, disponibilidad, variedad, oferta de complementarios, de paquetes y nuevos productos y recomendación de similares.

b. Precio: información de precios, moneda de transacción, convertidor de moneda, opción de comparar precios, mención de precio competitivo, descuentos, métodos de pago y gastos de envío.

c. Plaza: sitio web propio o desde intermediarios, presencia en sitios web de terceros, motores de búsqueda, relacionamiento y localización

d. Promoción: aplicaciones, e-RP en redes sociales y otros medios, marketing en motores de búsqueda, publicidad gráfica interactiva, correo electrónico, mensajes de texto

3 Los nombres de los autoservicios han sido cambiados para proteger su real identidad. en dispositivos móviles, promoción de ventas y marketing directo.

e. Servicio al cliente: disponibilidad de medios y horarios, tiempo de respuesta a correos, seguimiento a entrega del producto, reclamaciones, sugerencias o peticiones y existencia de políticas.

f. Plataforma: calidad del sistema representada por usabilidad, accesibilidad, velocidad de cargue, navegabilidad, confiabilidad; diseño del sitio web o estética representado por diseño visual, personalidad, estilo, redacción de textos, marca, gráficos, color, tipografía, resolución y tamaño de pantalla; estructura del sitio web o arquitectura de la información representada por encabezados y pies de páginas, plantillas, categorías de productos, menú, buscador, registro y área del usuario, información de la organización, mapa del sitio web, página de transacción; contenido representado por actualización, información de interés e intercambio de información entre usuarios.

A continuación, se presenta la evaluación realizada a la mezcla de mercadeo digital presente en las plataformas de venta virtual de autoservicios en Colombia.

La plataforma Fontana.com presenta una muy buena conformación en relación con: a) producto, con un puntaje de 3,8 (descripción a profundidad de su oferta e imágenes nítidas ampliables); b) precio, con un puntaje de 3,8 (precio actual y con rebaja, variados 
métodos de pago e información clara sobre costos de envío); c) plaza, con un puntaje de 4,2 (amplia cobertura nacional, buenos tiempos de entrega y aplicación); d) servicio al cliente, con un puntaje de 3,8 (chat, PQR y política de compra informada); y e) plataforma, con un puntaje de 3,9 (usabilidad, centro de ayuda, información del proceso de compra, posicionamiento, tiempo de carga, navegabilidad, intuitividad, certificados de seguridad, tratamiento de datos, estética y desarrollo de marca coherente, buena estructura de la arquitectura web, plantillas consistentes, categorización de productos, contenido actualizado de interés). Se observan debilidades en aspectos como la promoción, con un puntaje de 2,5 (véase figura 1).
La plataforma Kolmena.com presenta una muy buena conformación en relación con: a) producto, con un puntaje de 3,7 (descripción de su oferta e imágenes nítidas ampliables); b) precio, con un puntaje de 3,0 (precio actual y con rebaja, variados métodos de pago e información clara sobre costos de envío); c) plaza, con un puntaje de 3,4 (amplia cobertura nacional); d) servicio al cliente, con un puntaje de 3,4 (PQR y política de compra informada); y e) plataforma, con un puntaje de 3,8 (usabilidad, sección con preguntas frecuentes, información del proceso de compra, posicionamiento, tiempo de carga, navegabilidad, intuitividad, certificados de seguridad, tratamiento de datos, estética y desarrollo de marca coherente, buena estructura de la

Figura 1. Mezcla de mercadeo digital y plataforma Fontana.com.

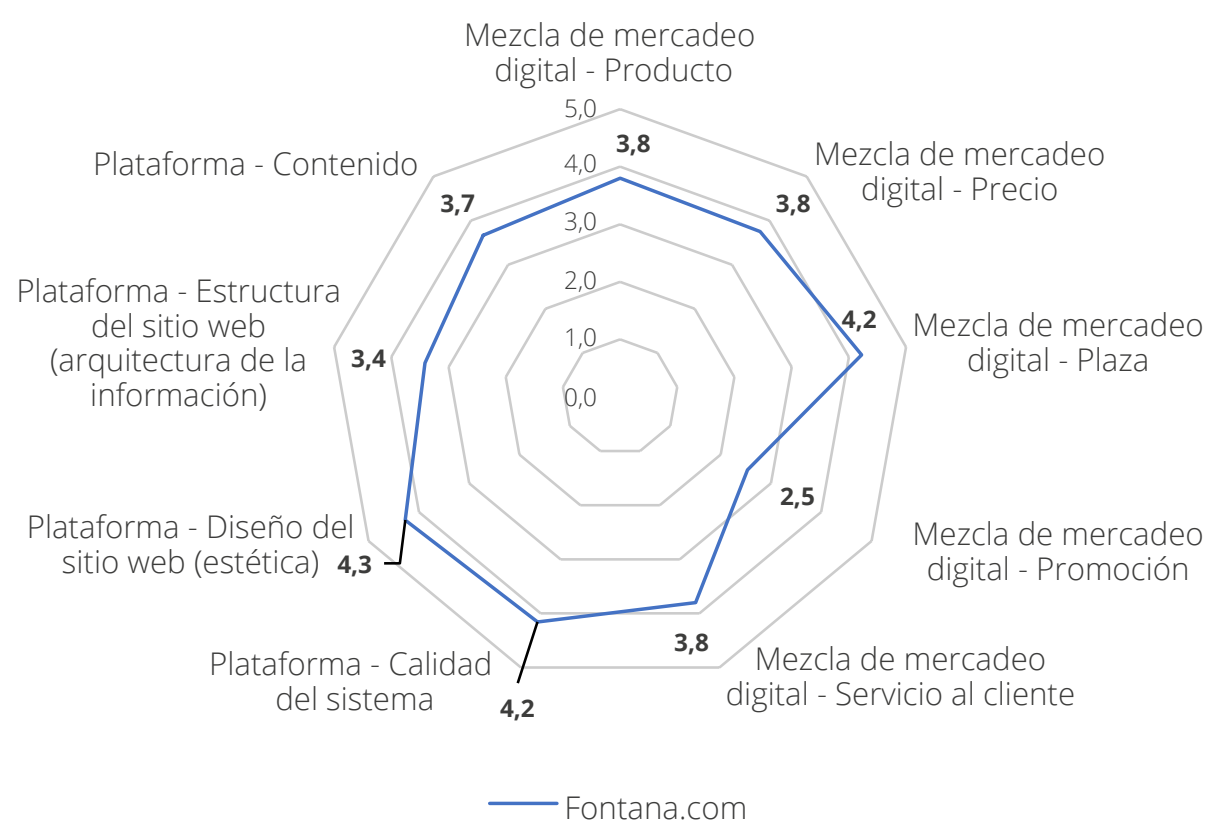

Fuente: elaboración propia de las autoras. 
arquitectura web, plantillas consistentes, categorización de productos y contenido actualizado de interés). Se observan debilidades en aspectos como la promoción, con un puntaje de 2,7 , y del contenido del sitio web, con un puntaje del 2,7 (véase figura 2).

La plataforma Mandala.com presenta una buena conformación en relación con: a) plaza, con un puntaje de 3,3 (amplia cobertura nacional y gran cantidad de visitas a su sitio web a nivel nacional); y b) servicio al cliente, con un puntaje de 3,0 (chat, seguimiento a pedidos, $\mathrm{PQR}$ y política de compra informada). Se observan debilidades en aspectos como el producto, con un puntaje de 2,2; el precio, con un puntaje de 2,5; la promoción, con un puntaje 1,8; la calidad del sistema, con un puntaje de 2,8; y el contenido del sitio web, con un puntaje de 1,7 (véase figura 3).

La plataforma Dominico.com presenta una deficiente conformación en relación con: a) plaza, con un puntaje de 3,9 (amplia cobertura nacional); y b) plataforma, con un puntaje de 3,7 (usabilidad, centro de ayuda, información del proceso de compra, tiempo de carga, navegabilidad, intuitividad, certificados de seguridad, desarrollo de marca coherente, buena estructura de la arquitectura web, categorización de productos y contenido actualizado de interés). Se observan debilidades en aspectos como el producto, con un puntaje de 2,9; el precio, con un puntaje de 2,9; la promoción, con un puntaje de 2,7; y el servicio al cliente, con un puntaje de 2,6 (véase figura 4).

Figura 2. Mezcla de mercadeo digital y plataforma Kolmena.com.

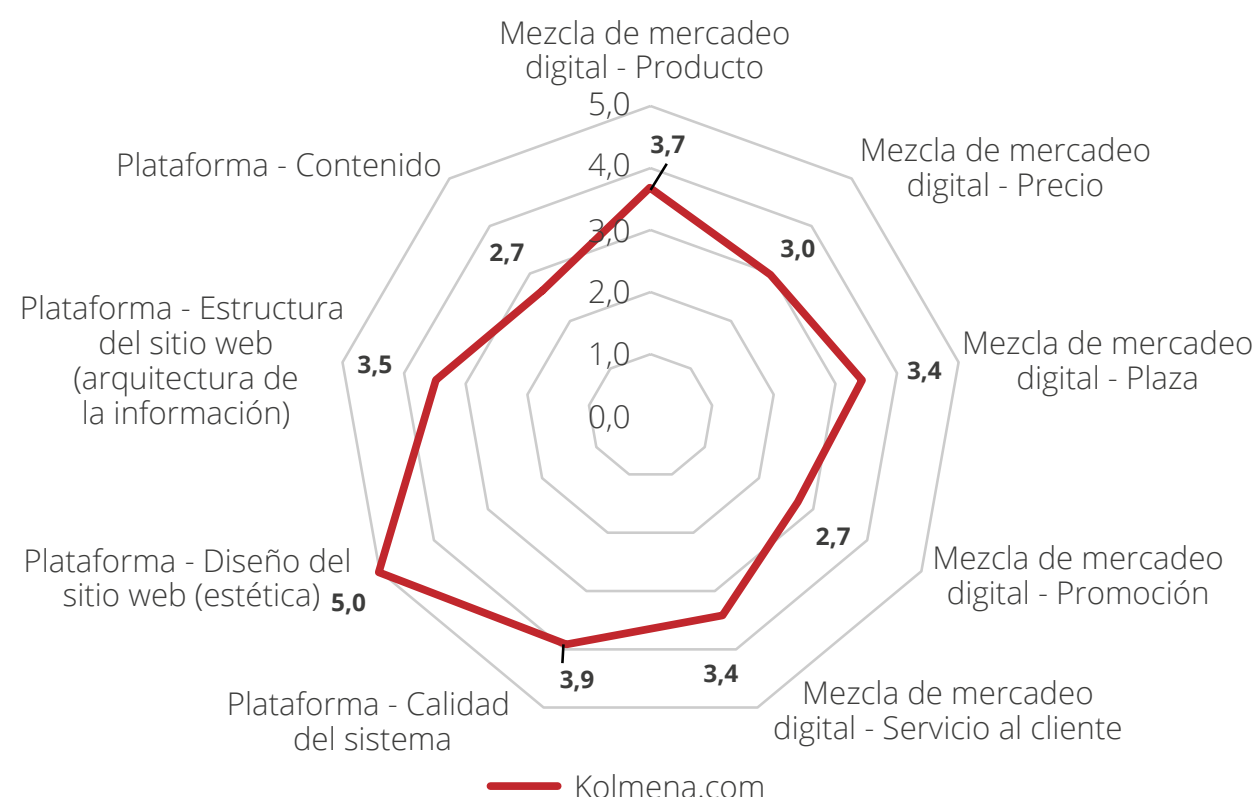

Fuente: elaboración propia de las autoras. 
Figura 3. Mezcla de mercadeo digital y plataforma Mandala.com.

\section{Mezcla de mercadeo \\ digital - Producto}

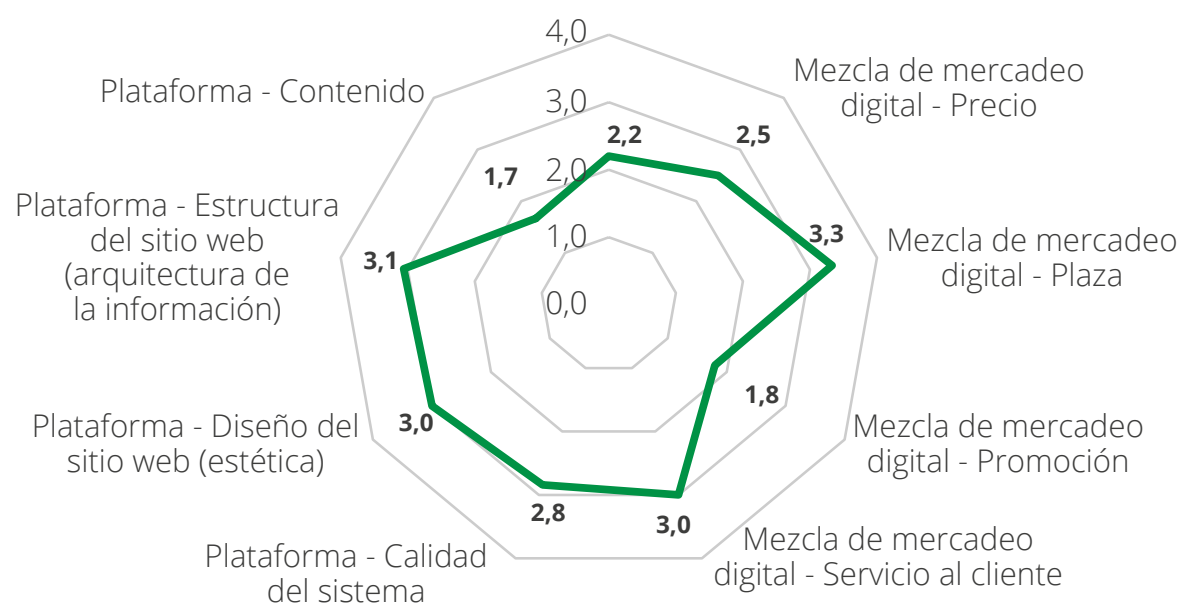

del sistema

Mandala.com

Fuente: elaboración propia de las autoras.

Figura 4. Mezcla de mercadeo digital y plataforma Dominico.com. Fuente: elaboración propia de las autoras.

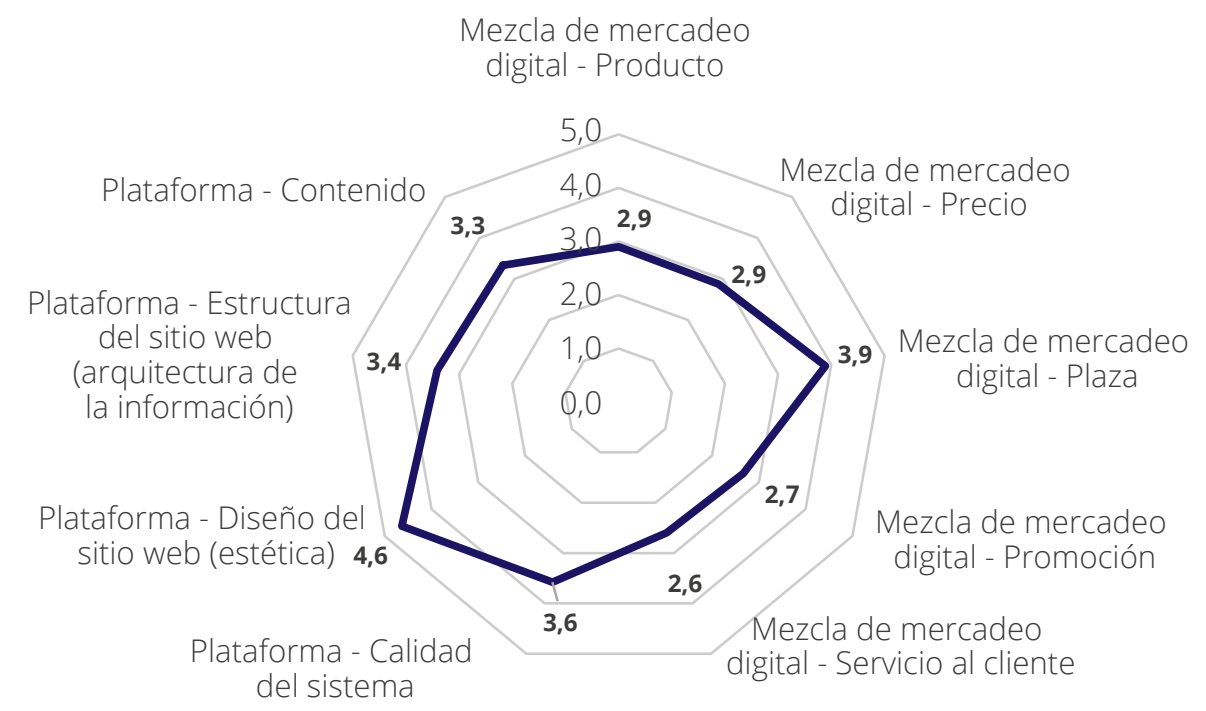

Dominico.com

Fuente: elaboración propia de las autoras. 


\section{Discusión}

Como puede observarse, las mejores plataformas de venta virtual de autoservicios en cuanto a su estructuración son: Fontana.com (primer lugar), Kolmena.com (segundo lugar) y Dominico.com (tercer lugar). La plataforma de venta virtual de Mandala.com presenta diversas deficiencias en aspectos sensibles que requieren atención para poder aprovechar todas las potencialidades que el comercio electrónico ofrece.

La implementación de la mezcla de mercadeo digital en estos sitios web, que son referente en el país, ha venido teniendo una mejora muy considerable en los últimos tiempos, en aspectos como: a) la presentación del producto, en la que se proporcionan todas sus características visuales y técnicas, que han hecho que esta sea casi tan palpable como en la presencialidad; b) la política de precios, que es mucho más clara y transparente para el cliente y los diferentes métodos de pago que facilitan las transacciones al usuario; c) la variada disponibilidad de canales de entrega del producto y la interrelación entre estos; d) la estrecha cooperación con las redes sociales, marketing en motores de búsqueda y los variados métodos de promoción aprovechados; e) el mejoramiento en los niveles del servicio al cliente, aunque existen aspectos por mejorar; y f) la estructuración sólida en las plataformas en cuanto a calidad técnica, diseño o estética, arquitectura de la información y contenido de interés para el cliente.
Las implementaciones en cuanto a la mezcla de mercadeo digital (producto, precio, plaza, promoción, servicio al cliente y plataforma) realizadas en estas plataformas de venta virtual tienen muy en cuenta las problemáticas detectadas y hacen un esfuerzo sistemático y sostenido por: a) ofrecer una infraestructura tecnológica robusta, fácil de utilizar y que esté al servicio del cliente; b) ofrecer variados métodos de pagos; c) aumentar los niveles de confianza en el manejo de datos personales y financieros; d) crear una experiencia positiva y agradable en todos los puntos de contacto con el cliente; e) ofrecer diferentes métodos de contacto; f) disponer de información de interés para los usuarios; e g) informar al usuario sobre las políticas de compra.

La implementación de sitios web con una correcta estructuración de la mezcla de mercadeo digital es de vital importancia para tener claridad sobre los elementos por la que está compuesta y cómo están representados en el medio online para poder brindar una experiencia completa al cliente, toda vez que estamos hablando de un contexto de aplicación diferente al del marketing tradicional, lo cual facilita su reproducibilidad por parte de otras organizaciones que consideren tener presencia digital.

\section{Conclusiones}

Las plataformas de venta virtual de los autoservicios con presencia nacional han impulsado grandemente al subsector del comercio minorista. Estas se caracterizan por ser robustas, estar bien estructuradas en materia 
de mercadeo digital y crear una experiencia única para el cliente.

La revisión de las teorías en relación con la mezcla de mercadeo digital y de los modelos existentes para la evaluación de plataformas de venta virtual permite recapitular las bases del marketing tradicional e identificar las principales variables que intervienen en ella (categorías, subcategorías y variables) dentro del contexto virtual, para que así las empresas puedan tenerlas en cuenta en una posible implementación de la estrategia online.

Es claramente observable que las problemáticas que presentaban las plataformas de venta virtual en sus inicios han sido superadas para poner a disposición del usuario una infraestructura técnica amigable, intuitiva y de fácil uso, aproximación muy cercana al producto tangible, diversidad en los métodos de contacto y pago y variedad en los canales marketing y de promoción. Esto crea altos niveles de relacionamiento con el cliente y una experiencia completa para este durante todo el proceso de compra.

Esta investigación abre un camino para la estructuración de un modelo que permita medir las categorías, subcategorías y variables de la mezcla del mercadeo digital que deben estar presentes en las plataformas de venta virtual.

\section{Referencias}

Acosta Herrera, D., \& Martínez Bohórquez, A. (2018). Marketing Digital y su evolución en Colombia. Universidad Li- bre de Colombia. Consultado en abril de 2020. https://bit.ly/3/4MeuR

Asociación Española de Comercio Electrónico y Marketing Relacional (AECEM). (2009). Libro blanco del comercio electrónico. Guía práctica de comercio electrónico para PYMES. AECEM-FECEMD. https://libros.metabiblioteca.org/bitstream/001/243/8/ AECEM_Libro_Blanco.pdf

Allen, E., \& Fjermestad, J. (2002). E-Commerce Strategies: The Manufacturer Retailer Consumer Relationship. Americas Conference on Information Systems (AMCIS). http://aisel.aisnet.org/ amcis2000/67

Andréu, J. (2018). Las técnicas de análisis de contenido: Una revisión actualizada. Mastor.cl. Consultado en noviembre de 2016. https://bit.ly/3gkdWQr

Azadi, S., \& Rahimzadeh, E. (2012). Developing marketing strategy for electronic business by ussing McCarthy's four marketing mix model and Porter's five competitive forces. Emerging Market Journal, 2(2), 47-53. https://doi. org/10.5195/emaj.2012.25

Barnes, S. J., \& Vidgen, R. (2002). An integrative approach to the assessment of e-commerce quality. Journal of Electronic Commerce Research, 3(3), 114127. https://bit.ly/2EiwsMh

Bizhanova, K., Mamyrbekov, A., Umarov, I., Orazymbetova, A., \& Khairullaeva, A. (2019). Impact of digital marketing development on entrepreneurship. E3S Web of Conferences, 135(04023). https://doi.org/10.1051/ e3sconf/201913504023

Cámara Colombiana de Comercio Electrónico, Centro Nacional de Consultoría. (2019). Medición de indicadores 
-Tendencia de la oferta de Bienes y Servicios en línea. https://bit.ly/31 n5Dz2

Chaffey, D., \& Ellis-Chadwick, F. (2014). Marketing digital, estrategia, implementación y práctica. Pearson Educación.

Chaffey, D., \& Smith, P. (2013). EMarketing Excellence: Planning and Optimizing your Digital Marketing. Routledge.

Chaffey, D., Ellis-Chadwick, F., Johnston, K., \& Mayer, R. (2006). Internet Marketing. Strategy, Implementation and Practice. Harlow. Pearson Education.

Consejo Nacional de Política Económica y Social República de Colombia. (2009). Documento Conpes 3620. Lineamientos de política para el desarrollo e impulso del comercio electrónico en Colombia. https://bit.ly/317kx4u

Constantinides, E. (2002). The 4S Web-Marketing Mix model. Electronic Commerce Research and Applications, 1(1), 58-60. https://doi.org/10.1016/ S1567-4223(02)00006-6

González, O. R., Bañeguil T. M., Buenadicha M. (2013). El índice cuantitativo de calidad web como instrumento objetivo de medición de la calidad de sitios web corporativos. Investigaciones Europeas de Dirección y Economía de la Empresa, 19(1), 16-30. https://doi. org/10.1016/j.iedee.2012.07.004

Hernández, R. F. (2014). Metodología de la Investigación (6. ${ }^{\mathrm{a}}$ ed.). McGraw Hill Education.

Kannan, P. K., \& Li, H. (2016). Digital marketing: A framework, review and research agenda. International Journal of Research in Marketing, 34(1), 22-45. https://doi.org/10.1016/j.ijresmar.2016.11.006
Las transacciones digitales representaron $8,5 \%$ del PIB de Colombia para 2018. (1º de agosto de 2019). La República. https://bit.ly/2YtMDgu

Liacono, E. T., Watson R. T., \& Goodhue D. L. (2002). WEBQUAL'M: A measure of website quality. En K. Evans (ed.), Marketing Theory and Applications (vol. 13, pp. 432-438). American Marketing Association. https://bit.ly/3gqgzjM

Miranda, F. B., Barriuso, M. C., \& Cortés, R. (2005, 19 de septiembre). La banca por internet en España. Aplicación del índice de Evaluación (IEW) [boletín n. ${ }^{\circ}$ 2855]. Boletín Económico de ICE. https://bit.ly/2EcgcfU

Observatorio e-Commerce Colombia. (2016). 4to Estudio Transacciones Digitales: eCommerce \& Recaudo Colombia 2016 - Primer semestre 2017 [infografía]. https://bit.ly/2YIzOuj

Observatorio eCommerce Colombia. (2019). Medición de Indicadores de consumo del Observatorio eCommerce. https://bit.ly/3j3q2iT

Observatorio eCommerce. (2018). Transacciones Digitales Colombia: eCommerce \& Recaudo 2016/2017. https:// bit.ly/3hooDCW

Olsina, L., Rossi, G. (2002). A quantitative method for quality evaluation of web sites and applications. IEEE Multimedia, 9(4), 20-29. https://bit.ly/32j219Z

Park, Y., Gretzel, U. (2007). Sucess factors for destination marketing web sites: A quantitative meta-analysis. Journal of Travel Research, 46(1), 46-63. https:// doi.org/10.1177/0047287507302381

Polillo, R. (2011). Quality model for web (2.0) sites: A methodological approach and a proposal. En A. Harth \& N Koch 
(eds.), Current Trends in Web Engineering. ICWE 2011. Lectures Notes in Computer Science (vol. 7059). Springer. https://doi.org/10.1007/978-3-64227997-3_25

Portal ISO 25000. (2016). ISO/IEC 25010. https://bit.ly/32jtaAs

Universidad Nacional Abierta y a Distancia. (2016). Estándares y modelos de calidad del software. Obtenido de http://datateca.unad.edu.co/contenidos/301569/AVA_2014_II_-_301569/ AVA_ESTANDARES_Y_MODELOS_DE_ CALIDAD_DEL_SOFTWARE.pdf
Zeithaml, V., Parasuraman, A., \& Malhotra, A. (2002). Service quality delivery through web sites: A critical review of extant knowledge. Journal of the Academy of Marketing Science, 30(49), 362. https://doi. org/10.1177/009207002236911 